\title{
Benchmarking entre sistemas regionales de innovación: el caso de Santander y Antioquia, Colombia
}

\section{Benchmarking between regional innovation systems: the case of Santander and Antioquia, Colombia}

\author{
Claudia Patricia Cote-Peña ${ }^{1}$ \\ Claudia Patricia Meneses-Amaya² \\ Cristian Johan Arenas-Morantes ${ }^{3}$ \\ Danitza Ivonne Caballero-Pérez ${ }^{4}$
}

Recibido: diciembre 16 de 2015

Aceptado: abril 09 de 2016

\section{Resumen}

Este artículo analiza el actuar de dos importantes departamentos de la República de Colombia en materia de Ciencia, Tecnología e Innovación, CTI. Para ello, se utiliza un enfoque metodológico de tipo analítico-descriptivo y se implementa un análisis comparativo o benchmarking entre los Sistemas Regionales de Innovación, SRI, de Santander y Antioquia. Este trabajo pretende, además de contribuir a la construcción de una perspectiva actual de los SRI estudiados, orientar mediante la identificación de fortalezas y debilidades, el diseño y creación de políticas o programas que impulsen los procesos de generación y difusión del conocimiento en el departamento de Santander. Los resultados muestran que Santander presenta un rezago importante en materia de CTI con respecto a Antioquia, esto como reflejo de la limitada participación del sector productivo en el fortalecimiento de las capacidades de innovación, la desigual participación de los actores en iniciativas de CTI y los bajos niveles de inversión en Actividades de Ciencia, Tecnología e Innovación. Se recomienda que Santander trabaje en algunos aspectos fundamentales para la consecución de mejores resultados en los procesos de innovación departamental.

Palabras clave: benchmarking, Sistemas Regionales de Innovación, conocimiento, Ciencia, Tecnología e Innovación.

\begin{abstract}
This article analyzes the performance of two important states of Colombia in terms of Science, Technology, and Innovation, STI. To achieve this, a descriptive-analytical methodology is used and a comparative analysis, or benchmarking, is implemented between the Regional Innovation System, RIS, from Santander and the RIS from Antioquia. This work aims not only to contribute to the creation of a current perspective of the RIS studied, but also to guide through the strengths and weaknesses to a creation and design of policies or programs that encourage the processes of generation and dissemination of knowledge in the state of Santander. The results show that Santander presents a significant lag in terms of STI with respect to Antioquia. This is a reflection of the limited participation of the productive sector in the strengthening of innovation capabilities, unequal participation of stakeholders in initiatives of STI, and low levels of Activities of Investment in Science, Technology, and Innovation. It is recommended that Santander works on some important aspects to achieve better results in the processes of innovation in all the state.
\end{abstract}

Keywords: benchmarking, Regional Innovation Systems, knowledge, Science, Technology and Innovation.

1 Economista, Especialista en Alta Gerencia y en Dirección de Empresas, Universidad Industrial de Santander, Colombia.

E-mail: ccote@uis.edu.co

2 Economista, Universidad Industrial de Santander, Colombia. E-mail: claudia.meneses@correo.uis.edu.co

3 Estudiante de Economía, Universidad Industrial de Santander, Colombia. E-mail: cristianmorantes@hotmail.com

4 Estudiante de Economía, Universidad Industrial de Santander, Colombia. E-mail: danii1804@live.com 


\section{Introducción}

En el marco de la Economía Basada en el Conocimiento y bajo los nuevos esquemas de la globalización, las políticas de Ciencia, Tecnología e Innovación, CTI, han logrado consolidarse como un instrumento estratégico para transitar hacia un sendero de desarrollo sustentable (Arias, Arenas, Carrillo, \& Flórez, 2013). Sin embargo, estas políticas solo serán fructíferas si se cimientan en información veraz que denote el estado actual del territorio. Así, Oquendo-Gómez y Acevedo-Álvarez (2012) afirman que el desempeño de un Sistema de Innovación depende en gran parte de la información presente en el entorno.

Lo anterior refleja la importancia de contar con un sistema de información sólido que relacione a cada una de las instituciones partícipes en el Sistema de Innovación. Desde esta perspectiva, y para el caso concreto de Santander, la falta de información consolidada genera desconocimiento de la realidad actual de las iniciativas propias de la región y de las características de su Sistema Regional de Innovación, SRI (Arias et al., 2013). Por tal motivo, en este artículo se realiza un análisis descriptivo y comparado del estado actual del Sistema Regional de Innovación del departamento de Santander. Para ello se utiliza un enfoque metodológico de tipo analítico-descriptivo y se implementa un análisis comparativo o benchmarking entre los SRI de Santander y Antioquia. Lo anterior con el propósito de identificar fortalezas y debilidades del SRI de Santander frente al de Antioquia, de tal manera que orienten la toma de decisiones en materia de CTI.

Es preciso señalar que en la selección del SRI de referencia o benchmark se tuvo en cuenta la posición que ocupa Antioquia como departamento líder en competitividad, la similitud de aspectos socioeconómicos de este con el departamento de Santander, la presencia de capacidades científicas consolidadas, el avance en la creación y consolidación de escenarios de apoyo a la innovación, así como de estructuras de articulación presentes en el entorno antioqueño (Llisterri \& Pietrobelli, 2011). Todo lo anterior, sumado a la distinción que obtuvo Medellín como la ciudad más innovadora del mundo en el marco del concurso City of The Year (Camargo, 2013), justifica la elaboración de un análisis comparado entre los Sistemas Regionales de Innovación de Santander y Antioquia.

La importancia de este estudio radica en que puede servir para orientar el diseño y creación de políticas o programas de I+D que impulsen la generación de conocimiento, además de incentivar la inversión en CTI por parte de los actores que conforman el SRI de Santander. De esta forma, este artículo responde al cuestionamiento sobre ¿cuál es el estado actual del Sistema Regional de Innovación de Santander comparado con el Sistema Regional de Innovación de Antioquia? y se estructura en cuatro secciones. Seguido de esta introducción, se exponen los materiales y métodos utilizados. Luego, se presentan los resultados y discusión sobre el análisis comparativo entre el SRI de Santander y Antioquia, para finalizar con las principales conclusiones.

\section{Materiales y métodos}

La metodología del Benchmarking utilizada en este estudio ha sido adaptada como herramienta de evaluación y diagnóstico de Sistemas de Innovación. Su objetivo consiste en permitir la comparación de los diversos elementos que conforman un SRI y su respectivo funcionamiento con la manera en que estos son manejados por otros SRI de referencia que presentan un mejor desempeño, ofreciendo una visión general de su estructura y dinámica (Navarro-Arancegui, Franco-Rodríguez, Murciego-Alonso, \& Gibaja-Martíns, 2012). Bajo esta perspectiva, Zuluaga (2006) propone un esquema general en el que siguiendo una serie de etapas, es posible analizar los SRI mediante benchmarking. Estas etapas fueron implementadas en este estudio y se describen a continuación:

i) Definir el área, propósitos y objetivos del estudio: la delimitación del área de estudio tuvo en cuenta el 
perfil que expone Santander en el ámbito nacional e internacional como una región que cuenta con vocación para crear competencias basadas en CTI (Pinilla, Camacho, Guerrero, Pradilla, \& Cote, 2001), sumado a la motivación de construir una perspectiva clara del estado actual del SRI de Santander. Así, la implementación del benchmarking tiene como fin identificar, mediante un estudio detallado, las acciones que llevaron a Antioquia a consolidarse como departamento líder en competitividad.

ii) Determinar los Sistemas de Innovación a los cuales se va a estudiar: en el proceso de selección del SRI de referencia para el Benchmarking se recurrió al más reciente Escalafón de competitividad de los departamentos de Colombia (2015), el cual ubica a Antioquia en la posición de líder nacional en competitividad (Ramírez \& De Aguas, 2015). De esta forma, el liderazgo en competitividad ejercido por Antioquia, la similitud de aspectos socioeconómicos entre Antioquia y Santander, la presencia de capacidades científicas consolidadas, el avance en la creación y consolidación de escenarios de apoyo a la innovación (Llisterri \& Pietrobelli, 2011), sumado a la distinción que obtuvo Medellín como la ciudad más innovadora del mundo en el marco del concurso City of The Year, organizado por The Wall Street Journaly Citigroup (Camargo, 2013), justifican la elaboración de un análisis comparado entre los Sistemas Regionales de Innovación de Santander y Antioquia.

iii) Desarrollar un método para la recogida de datos: la información para este estudio se tomó de Informes Anuales del Observatorio Colombiano de
Ciencia y Tecnología, OCyT, Informes de Competitividad de la Cámara de Comercio de Bucaramanga, así como de documentos de acceso público del Ministerio de Educación Nacional y del Departamento Administrativo de Ciencia, Tecnología e Innovación, Colciencias. Para la elección de dichos documentos se tuvo en cuenta el grado de confiabilidad de las organizaciones que los desarrollaron.

iv) Selección y análisis de variables: la selección y definición de las variables se realizó a partir de una exhaustiva revisión de los siguientes documentos: Sistemas Regionales de Innovación en América Latina (Llisterri \& Pietrobelli, 2011), Informe Anual del Observatorio Colombiano de Ciencia y Tecnología (Observatorio Colombiano de Ciencia y Tecnología [OCyT], 2014b) y Plan Estratégico Departamental de Ciencia, Tecnología e Innovación de Santander 2020 (Arenas-Díaz et al., 2013). De este modo, se reconoció como una variable transversal la inversión en Actividades de Ciencia, Tecnología e Innovación, ACTI, como porcentaje del Producto Interno Bruto Departamental, PIBD. Además se identificó un conjunto de variables que inciden en las capacidades de innovación departamental.

Para efectos de este estudio, se tuvo en cuenta la clasificación que Fernández-de Lucio y Castro-Martínez (1995) hacen de los actores que conforman un SRI, agrupándolos en subsistemas, los cuales se tomaron como referente para agrupar las variables. En la tabla 1 se relacionan los subsistemas y se detallan las variables más relevantes en la dinámica competitiva de los SRI, las cuales se analizan en la siguiente sección.

\section{Subsistema científico}

Número de Instituciones de Educación Superior, IES, por cada 100.000 habitantes

Contribución de las IES a la inversión en ACTI como porcentaje del PIBD

Número de investigadores por cada 100.000 habitantes

Número de grupos de investigación por cada IES

Número de revistas indexadas en Colciencias por IES

Número de patentes, modelos de utilidad y diseños industriales por IES 


\section{Subsistema productivo}

Contribución de las empresas a la inversión en ACTI como porcentaje del PIBD

Índice Doing Business

Tasa de emprendimiento empresarial

Número de marcas registradas por cada 1.000 empresas

\section{Subsistema facilitador}

Presencia de entidades especiales promotoras de CTI

Contribución de las entidades del Gobierno Central a la Inversión en ACTI como porcentaje del PIBD

Presupuesto del Programa Ondas según entidad territorial

Proyectos de CTI aprobados y financiados por Colciencias

\begin{tabular}{l} 
Subsistema tecnológico \\
\hline Presencia de Parques Tecnológicos \\
Presencia de Incubadoras de Empresas de Base Tecnológica \\
Presencia de Centros de Desarrollo Productivo \\
Presencia de Centros de Desarrollo Tecnológico \\
Presencia de Centros Regionales de Productividad
\end{tabular}

\section{Subsistema financiero}

Bancos de segundo piso o bancos de fomento

Valor de los desembolsos de Bancoldex como porcentaje del PIBD

Valor de créditos otorgados por Finagro como porcentaje del PIBD

Recursos colocados por Findeter como porcentaje del PIBD

Presencia de fondos de capital de riesgo

Presencia de cooperativas financieras

Presencia de cofinanciadoras

Tabla 1. Variables identificadas en cada subsistema, basado en Llisterri y Pietrobelli (2011), OCyT (2014b) y Arenas-Díaz et al. (2013).

\section{Resultados y discusión}

\subsection{Análisis comparado de los SRI de Santander y} Antioquia

Las inversiones en Actividades de Ciencia, Tecnología e Innovación, ACTI, buscan incentivar en los procesos de generación de conocimiento un comportamiento dinámico e interactivo que incida favorablemente en el crecimiento y desarrollo de una región. Bajo esta perspectiva, el OCyT (2014a), basado en el Manual de Frascati (Organización para la Cooperación y Desarrollo Económicos [OCDE], 2003), definió cinco tipos de ACTI: Activi- dades de I+D, Actividades de Formación, Actividades de Servicios, Actividades de Administración y Actividades de Innovación.

Ahora bien, de acuerdo con la figura 1, entre 2010 y 2014 la inversión en ACTI realizada por Antioquia y Santander no alcanzó el 1\% del PIB departamental. Cabe señalar que si bien Antioquia dedicó mayores recursos al desarrollo de estas actividades con respecto a Santander, para el año 2014 disminuyó su inversión en ACTI. Por su parte, en Santander se evidenció un incremento de 0,08 puntos porcentuales, al pasar del 0,13\% en 2010 a 0,21\% en 2014. 


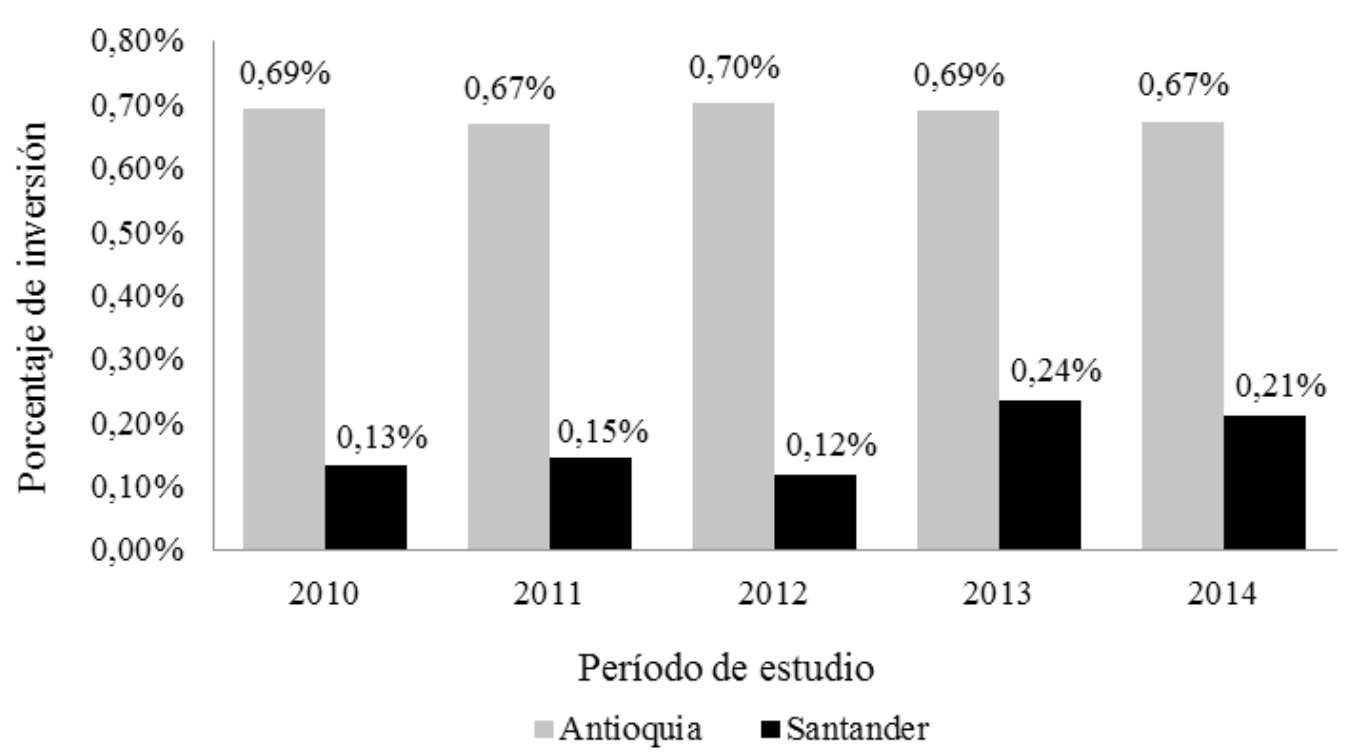

Figura 1. Inversión en ACTI como porcentaje del PIB departamental, 2010-2014. Modificado de OCyT (2014b).

\section{i) Subsistema científico}

La oferta de educación superior evidencia la fortaleza para formar individuos capaces de incidir de manera positiva en el desarrollo regional. En este sentido, la variable número de Instituciones de Educación Superior, IES, permitió identificar que en el año 2014 Antioquia contaba con 0,73 IES por cada 100.000 habitantes, mientras que Santander tenía
0,82 IES por cada 100.000 habitantes. Además, teniendo en cuenta la importancia de las IES, resulta interesante analizar la inversión que estas realizan en ACTI. La figura 2 muestra la destacada participación de las IES en Santander, las cuales alcanzaron niveles de inversión superiores al $80 \%$, demostrando que además de ser el actor que realiza mayores inversiones en ACTI, impulsa la dinámica innovadora del departamento. En lo que respecta a Antioquia, dicha inversión no superó el 30\%.

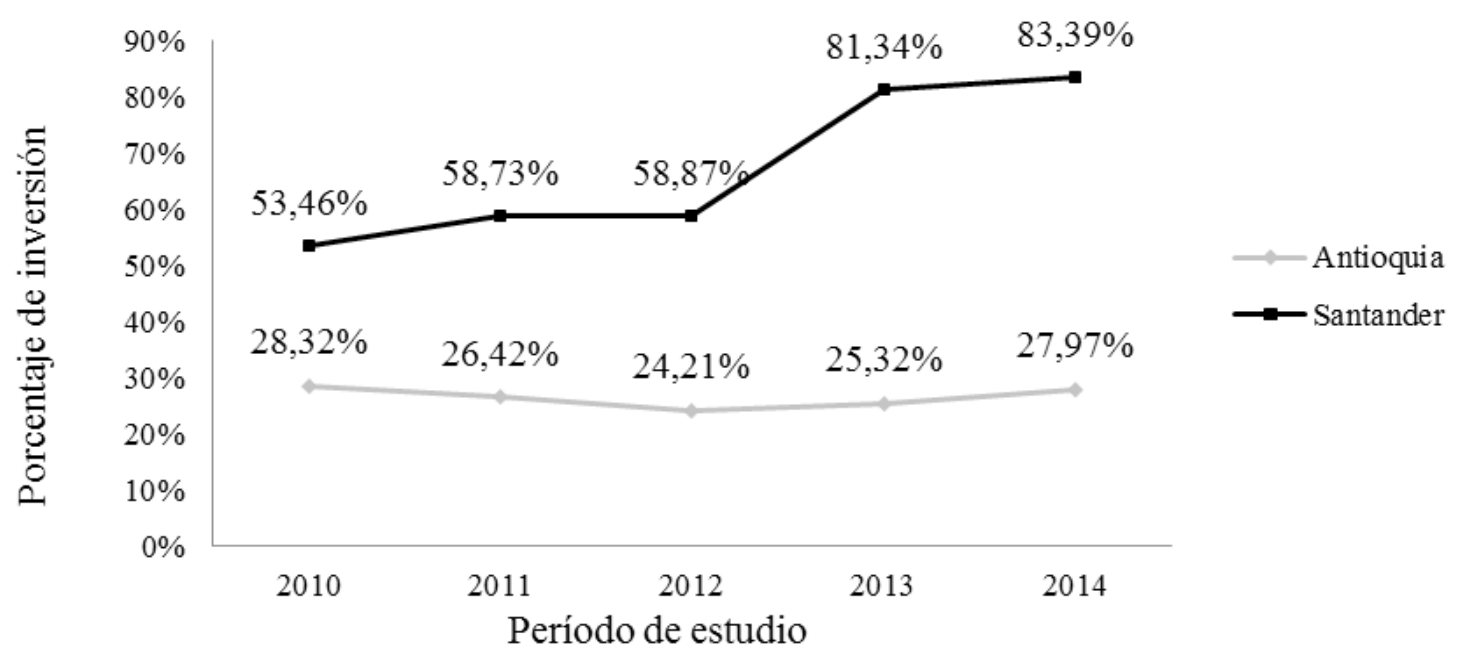

Figura 2. Contribución de las IES a la inversión en ACTI como porcentaje del PIB departamental, 2010-2014 (OCyT, 2014 b). 
Con respecto al número de investigadores, el departamento de Antioquia cuenta con una mayor población investigadora, presentando 26,52 investigadores por cada 100.000 habitantes. Por su parte, Santander tan solo alberga 18,92 investigadores por cada 100.000 habitantes. En cuanto a los grupos de investigación, se observa en Antioquia la presencia de 13,40 grupos de investigación por cada IES, dato similar al caso de Santander, en donde hay 12,20 grupos de investigación por cada IES.

Cabe mencionar que en el año 2014 se indexaron a Colciencias 73 revistas especializadas en CTI por parte de Antioquia y 25 en el caso de Santander. Al analizar esta información de acuerdo a las IES de cada departamento, se puede afirmar que Antioquia y Santander registraron 1,55 y 1,47 revistas por cada IES, respectivamente. En este sentido, se identificó la necesidad de incentivar la creación o acreditación de las revistas pertenecientes a la región santandereana, para así poder hacer visible los resultados de los procesos científicos y tecnológicos.

Adicionalmente, el número de patentes, modelos de utilidad y diseños industriales concedidos por la Superintendencia de Industria y Comercio en 2013, da cuenta de un mayor registro de producción intelectual por parte de Antioquia, lo que muestra más dinamismo en los procesos de generación y difusión del conocimiento. En la tabla 2 se puede observar el comportamiento de estas variables por departamento.

\begin{tabular}{lccc}
\hline Departamento & $\begin{array}{c}\text { Patentes de } \\
\text { invención }\end{array}$ & $\begin{array}{c}\text { Modelos de } \\
\text { utilidad }\end{array}$ & $\begin{array}{c}\text { Diseños } \\
\text { industriales }\end{array}$ \\
\hline Antioquia & 0,77 & 0,47 & 0,81 \\
Santander & 0,29 & 0,12 & 0,53 \\
\hline
\end{tabular}

Tabla 2. Número de patentes, modelos de utilidad y diseños industriales por cada IES, 2013 (OCyT, 2014b).

\section{ii) Subsistema productivo}

A partir de la información consignada en la figura 3 se puede inferir que el sector empresarial en Antioquia es quien le apuesta a la generación de capacidades innovadoras del departamento; muestra de ello es la participación de las empresas en la inversión en ACTI, que en el periodo 2010-2014 fue, en promedio, del 50\%. A diferencia de Antioquia, la inversión en ACTI realizadas por las empresas en Santander ha presentado una reducción sostenida en los últimos 5 años. Por consiguiente, para perfilarse como una región empresarial altamente competitiva, es necesario mejorar los niveles de inversión en actividades científico-tecnológicas que incrementen las innovaciones de las empresas. 


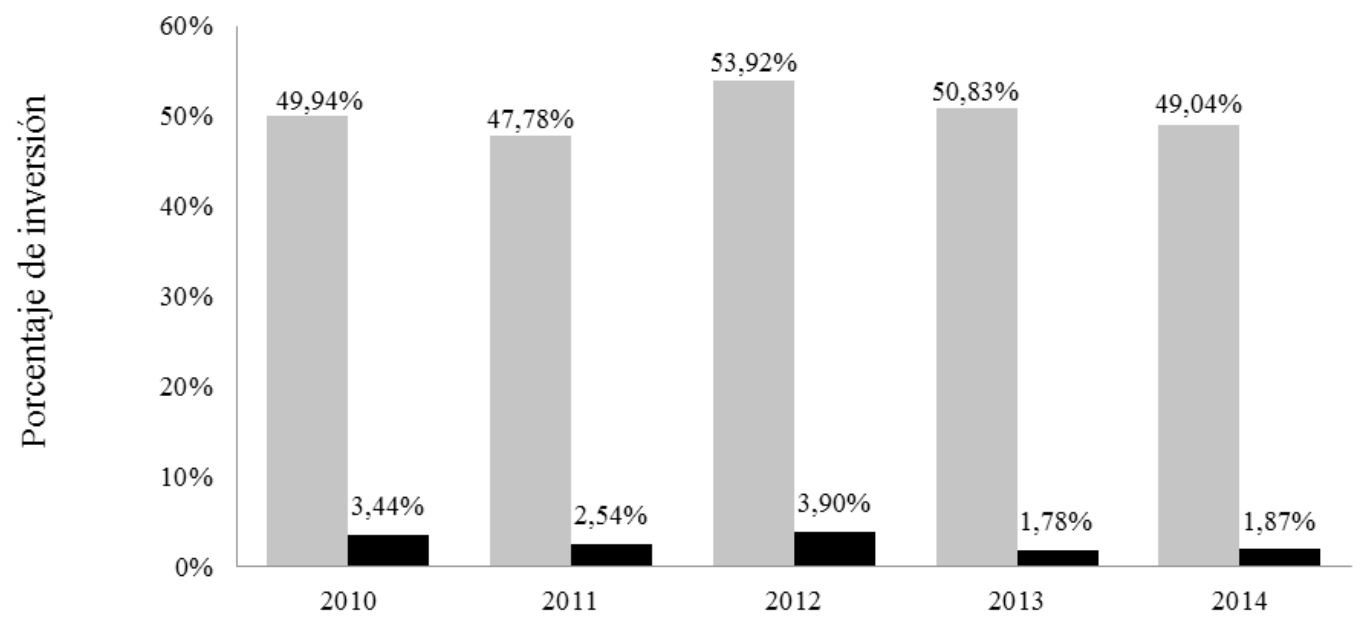

Período de estudio

Antioquia $\quad$ Santander

Figura 3. Contribución de las empresas a la inversión en ACTI como porcentaje del PIB departamental, 2010-2014 (OCyT, 2014b).

El Índice Doing Business, que mide la forma en que las regulaciones gubernamentales fomentan o restringen la actividad empresarial, ubica a Medellín y Bucaramanga en los puestos 11 y 14, respectivamente (Doing Businnes Subnational, 2013). Si bien este posicionamiento significa que en la capital antioqueña las condiciones para el desarrollo de una empresa se tornan más favorables, tanto Antioquia como Santander deben seguir fomentando un ambiente más propicio para el desarrollo de empresas, que le apuesten al fortalecimiento del SRI.

De igual forma, resultó de gran interés analizar la tasa de emprendimiento empresarial de estos departamentos. De este modo, se logró identificar que para 2014, en Antioquia y Santander, se constituyeron 50 y 88 empresas por cada 10.000 habitantes, respectivamente. Esta situación podría sugerir que Santander pretende mejorar las condiciones laborales y productivas del departamento. No obstante, es preciso señalar que si bien el departamento santandereano mostró una mejor dinámica de emprendimiento empresarial, la cancelación de las empresas también se registró en mayor proporción. Así, en 2014 la tasa de morta- lidad empresarial para Santander correspondió a 89 empresas por cada 10.000 habitantes, mientras que en Antioquia fue de 45 empresas por cada 10.000 habitantes (Cámara de Comercio de Bucaramanga, 2015).

Por último, y de acuerdo a la información de la Superintendencia de Industria y Comercio, de las 11.092 marcas otorgadas en el país durante el 2013, 2.461 correspondieron a Antioquia, lo que equivale a 17 marcas por cada 1.000 empresas, ubicándolo así en el primer lugar a nivel nacional. Entre tanto, a Santander le fueron concedidas 353 marcas, es decir, 5 por cada 1.000 empresas, ocupando el cuarto lugar a nivel nacional (Cámara de Comercio de Bucaramanga, 2014).

\section{iii) Subsistema facilitador}

En Colombia hacen parte del subsistema facilitador los siguientes actores: el Departamento Nacional de Planeación, DNP, el Departamento Administrativo de Ciencia, Tecnología e Innovación, Colciencias, el Consejo Nacional de Política Económica y Social, Conpes, el Observatorio Colom- 
biano de Ciencia y Tecnología, OCyT, y los Ministerios de la República. Además, los departamentos cuentan con gobernaciones, alcaldías, secretarías y demás órganos de articulación o entidades de interfaz, como son: los Consejos Departamentales de Ciencia, Tecnología e Innovación, CODECTI en Santander y CODECYT en Antioquia; los Comités Universidad Empresa Estado, CUEE en Antioquia y CUEES en Santander, y las Comisiones Regionales para la Competitividad, Comisión de Competitividad de Antioquia y Santander Competitivo.

Adicionalmente, Antioquia cuenta con otras entidades que imprimen un mayor dinamismo al SRI, como son: el Centro de Innovación y Negocios RUTA N, la fundación empresarial ProAntioquia, la corporación Tecnnova y el centro interactivo Par- que Explora-Acuario-Planetario. Ello demuestra el compromiso de Antioquia con la promoción de una cultura innovadora que incremente la competitividad, dinamice la economía y fortalezca las estrategias de CTI.

En la figura 4 se compara la inversión en ACTI realizada por la administración pública de cada departamento, observándose que en Santander la participación gubernamental en las ACTI ha disminuido en los últimos años. Si bien dicha inversión ha disminuido, es preciso resaltar que la inversión total en ACTI del departamento ha aumentado, lo cual obedece a que en el SRI santandereano la mayor contribución en ACTI es desarrollada por el subsistema científico.

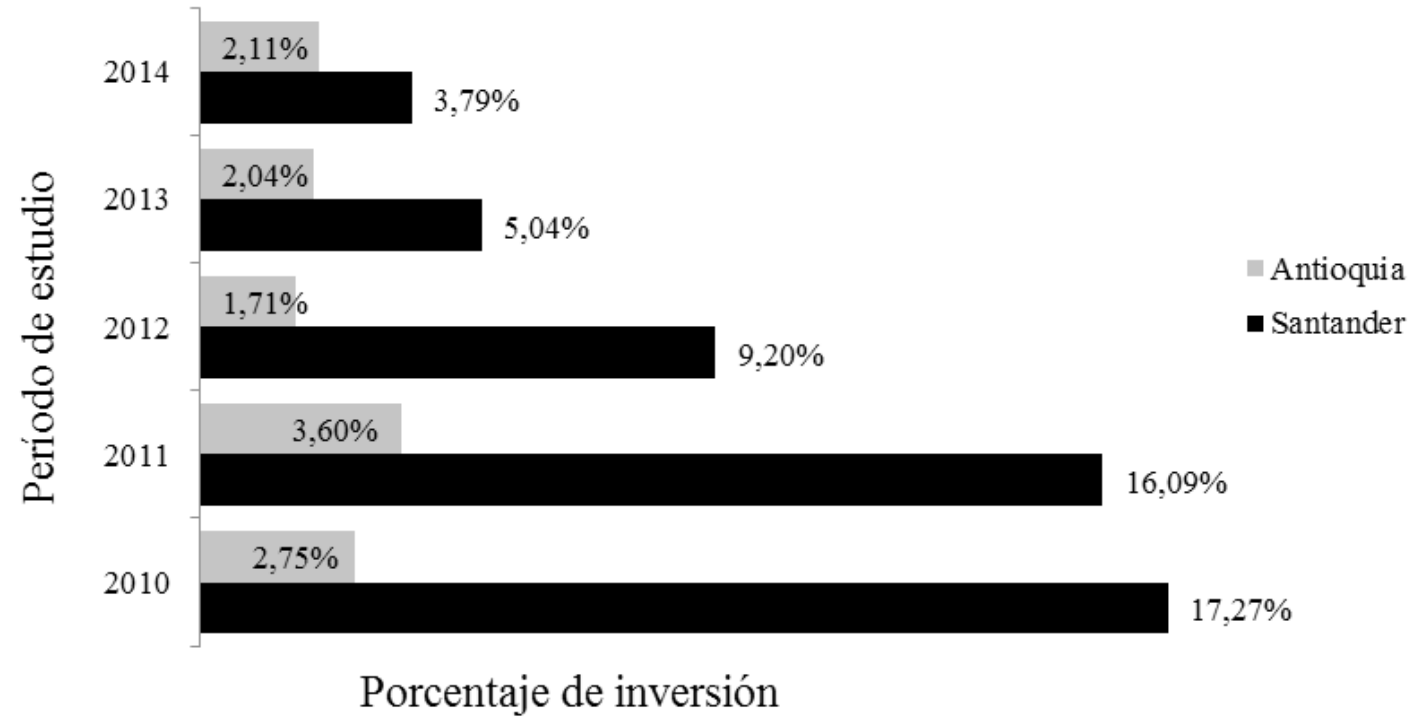

Figura 4. Contribución de las Entidades del Gobierno Central a la inversión en ACTI como porcentaje del PIB departamental, 2010-2014 (ОСуT, 2014b).

Por su parte, Colciencias fomenta una cultura ciudadana y democrática en materia de CTI liderando el Programa Ondas, el cual busca incentivar una cultura investigativa e innovadora desde la niñez. En este contexto, la inversión de este programa en 2013 tuvo una mayor participación en Santander, con un valor de $\$ 271.000 .000$ COP que correspondió al 0,22\% de la inversión departamental en
ACTI; mientras que en Antioquia la inversión fue de $\$ 176.000 .000$ COP, lo que representó un 0,027\% de la inversión departamental en ACTI.

Con referencia al apoyo para la generación de proyectos, entre 2010 y 2013, Colciencias aprobó y financió para Antioquia y Santander 590 y 346 proyectos en $\mathrm{CTI}$, respectivamente. Al analizar el 
número de proyectos aprobados y financiados de acuerdo a los grupos de investigación registrados en GrupLAC a 2013 en cada departamento, se encontró que en Antioquia, Colciencias financió 1,02 proyectos por cada grupo de investigación, mientras que en Santander fue de 1,42 proyectos por cada grupo. No obstante, al observar los recursos destinados a estos proyectos, se puede inferir que en Antioquia, los requerimientos para el desarrollo de los proyectos son superiores a Santander, pues mientras para Antioquia el monto de los mismos correspondió al 57,59\% del valor total financiado a nivel nacional, para Santander se destinó el 4,53\% del total nacional.

\section{iv) Subsistema tecnológico}

Uno de los principales actores que conforman este subsistema son los Parques Tecnológicos, PT. En Antioquia se encontró la participación de tres importantes PT: Parque Tecnológico del Software-Parquesoft de Antioquia; Parque del Emprendimiento de Medellín, Parque E; y el Parque Tecnológico Manantiales. En el caso de Santander se identificó el Parque Tecnológico de Guatiguará, PTG, considerado como uno de los proyectos urbanístico, tecnológico y empresarial con mayor avance relativo en el país. De igual manera, los dos departamentos cuentan con la presencia de TecnoParque, una red liderada por el Servicio Nacional de Aprendizaje, SENA.

Otro actor que incide en el entorno empresarial son las Incubadoras de Empresas de Base Tecnológica. En el departamento de Antioquia se ubican: la Corporación Incubadora de Empresas de Base Tecnológica del Oriente antioqueño, GÉNESIS; la Corporación Incubadora de Empresas de Base Tecnológica de Antioquia, IEBTA; la Corporación Incubadora de Agrupamientos Agroindustriales de Urabá, INCUBAR URABÁ; y el Centro Integral de Servicios Empresariales, CREAME. En Santander se evidencia la presencia de la Incubadora Corporación Bucaramanga Emprendedora, CBE.
Los Centros de Desarrollo Productivo, CDP, también desempeñan un importante papel dentro de este subsistema, puesto que a través de ellos se logra desarrollar procesos innovadores que mejoran la productividad y competitividad empresarial (Baena, Sánchez, \& Montoya, 2006). Bajo este contexto, en consideración al caso de Santander se identificaron el CDP de Joyería y el CDP de Confecciones, mientras que Antioquia carece de dichos centros.

Por su parte, los Centros de Desarrollo Tecnológico, $C D T$, enfocan sus actividades al dominio y generación de conocimientos especializados en tecnologías propias de un sector. En el caso de Antioquia, se destaca la existencia de siete CDT reconocidos por Colciencias: Centro de Innovación y Desarrollo Tecnológico País del Conocimiento, Corporación Calidad, Corporación Ecoeficiente, Instituto de Capacitación e Investigación del Plástico y del Caucho (ICIPC), Corporación Centro de la Ciencia y la Investigación Farmacéutica (CECIF), Corporación Centro de Investigación y Desarrollo Tecnológico del Sector Eléctrico (CIDET), Instituto de Ciencia y Tecnología Alimentaria (INTAL). Santander cuenta con tres CDT ubicados en el Parque Tecnológico de Guatiguará: el Centro de Desarrollo Tecnológico de Gas, la Corporación para la Investigación de la Corrosión $(\mathrm{ClC})$ y la Corporación para la Investigación y Desarrollo en Asfaltos en el Sector Transporte e Industrial (CORASFALTOS).

Se encuentran también en este subsistema los Centros Regionales de Productividad, CRP, los cuales además de promover la participación de los diferentes sectores productivos, son los encargados de divulgar regionalmente las líneas de fomento, las convocatorias y los incentivos a la innovación otorgados por Colciencias (Palacios-Preciado \& Duque-Oliva, 2011). Para el caso de Antioquia, se encontró el Centro de Ciencia y Tecnología de Antioquia (CTA), institución que convierte el conocimiento en herramientas para la generación del desarrollo económico y social, ofrece un portafolio de servicios dirigidos a tres líneas de acción; Productividad, Educación, Agua y Medio Ambiente. De igual manera, el Centro de Productividad y 
Competitividad del Oriente Colombiano, CPC, en el departamento de Santander, dirige sus actividades al mejoramiento de la productividad y competitividad regional mediante la gestión de proyectos de innovación, transferencia y apropiación de tecnologías.

\section{v) Subsistema financiero}

Entre los actores que hacen parte de este subsistema se encuentran los bancos de segundo piso o bancos de fomento, dentro de los cuales se destaca el Banco de Desarrollo Empresarial, Bancoldex. En 2013, este banco otorgó en créditos para Santander un valor cercano al $0,23 \%$ del PIB, mientras en el 2014 el valor ascendió al 0,26\%, esto destinado a capital de trabajo, modernización y otros servicios empresariales. Por otra parte, en Antioquia, para los mismos años, el valor correspondió al 0,54\% y $0,52 \%$, respectivamente. Ahora bien, el valor de los créditos otorgados en el año 2014 por Finagro correspondió al 1,09\% del PIB en Antioquia y a 0,91\% del PIB en Santander. Así mismo, entre el período 2010 y 2014 el monto de los recursos colocados por Findeter en Antioquia y Santander correspondió a $\$ 1.141 .695 .000$ COP y $\$ 1.148 .789 .000$ COP, respectivamente.

De igual forma, se identificaron los siguientes Fondos de Capital de Riesgo: Capital Medellín, Fondo de Capital de Riesgo de las Empresas Públicas de Medellín (EPM), Fondo de Capital de Riesgo Progresa Capital y Socialatom Ventures en Antioquia. Mientras que en Santander se encuentran el Fondo de Capital Privado Santander Inmobiliario y Corficolombiana (Fondo de Capital de Riesgo Kandeo Fund I). De lo anterior, se aprecia la ventaja con que cuenta Antioquia; de hecho, Medellín es considerada la capital de fondos de riesgo en Colombia.

Las Cooperativas Financieras identificadas para Antioquia son: Cooperativa Financiera de Antioquia (CFA), Coofinep, Confiar, Cotrafa, Cooperativa Financiera John F. Kennedy. En Santander hacen presencia la Cooperativa Multiactiva de Educado- res de Santander (COMULDESAN), la Financiera Comultrasan, Copacrédito, Comuldesa, Cooprofesores y la Cooperativa Energética de Ahorro y Crédito (FINECOOP). Por último, se identificó la presencia análoga en los dos departamentos de las siguientes Cofinanciadoras: Fondo Emprender-SENA, Bancoldex con INNpulsa Mipymes, Colciencias y Fundación de la Mujer.

3.2 Fortalezas y debilidades del Sistema de Innovación de Santander

\section{i) Fortalezas}

Los resultados del análisis comparado evidencian la fortaleza que posee Santander en cuanto a formación de capital humano con destacadas capacidades innovadoras. En este sentido, cabe destacar que la mayor presencia de IES por habitante en Santander, junto a los altos niveles de inversión realizada en ACTI por parte de las IES, son factores que contribuyen significativamente al desarrollo de habilidades y competencias en sus habitantes, lo cual incide de manera positiva en el crecimiento y desarrollo económico de la región.

De acuerdo con Canever y Carraro (2012), la constitución de nuevas empresas en un territorio incide directamente en el crecimiento económico del mismo, debido a que sirven como mecanismo de difusión del conocimiento, aumentan la competencia y la diversificación de bienes y servicios en una región. Bajo este contexto, y según los resultados del análisis, el emprendimiento empresarial se constituye como otra de las grandes fortalezas que posee el SRI de Santander, donde la tasa de emprendimiento registró la constitución de 88 empresas por cada 10.000 habitantes en 2014 ubicándolo como la tercera región del país con mayor tasa de emprendimiento empresarial. De esta manera, se pretende forjar una estructura de mercado más competitiva, en donde la complementariedad entre conocimiento e información incremente la actividad innovadora y el crecimiento económico de la región. 
Así mismo, es de suma relevancia y constituye una fortaleza para el SRI de Santander, contar con entidades que apoyan, coordinan e integran los procesos de CTI, como lo son: las Entidades Gubernamentales, quienes realizan importantes inversiones en Actividades de Ciencia, Tecnología e Innovación; Colciencias, quien a través del Programa Ondas fomenta la formación de capacidades en CTI, las cuales al ser vinculadas con el tejido social y productivo favorecen la innovación y la competitividad del departamento.

Bajo este mismo contexto, es preciso resaltar la importancia del Observatorio Colombiano de Ciencia y Tecnología para el SRI como entidad generadora de conocimiento, que integra, interpreta y difunde las estadísticas, constituyendo así una fuente de información que apoya el fortalecimiento de las capacidades para la construcción, uso y apropiación de los distintos indicadores de CTI.

Del mismo modo, se identificó como fortaleza la presencia activa de espacios que tienen como principal objetivo afianzar el desarrollo tecnológico de las microempresas mediante la atención en: diseño, organización de la producción, formación administrativa, control de calidad y comercialización. En este sentido, los Centros de Desarrollo Productivo de Joyería y de Confecciones desempeñan un importante papel en la adecuada tecnificación de procesos productivos que mejoran la competitividad empresarial, lo que a su vez genera bienestar para las comunidades o sectores vinculados, así como un desarrollo económico y social para la región.

Si bien las variables analizadas en el apartado anterior evidencian que cada departamento de estudio posee un único Centro Regional de Productividad, un aspecto clave para Santander es contar con el Centro de Productividad y Competitividad del Oriente como articulador entre oferentes y demandantes de tecnología, el cual tiene por objeto propender por el mejoramiento de la productividad y competitividad de las empresas a nivel regional, mediante la gestión pública y privada de proyectos de innovación, transferencia y apropiación de tecnologías.

\section{ii) Debilidades}

Gracias al adecuado desempeño de varios actores que conforman el SRI, Santander ha logrado avanzar en ciertos aspectos; no obstante, existen diversos factores que lo afectan negativamente. En primer lugar, se hace referencia a la falta de información disponible y actualizada por parte de algunos actores del SRI, hecho que dificultó la recopilación de la información. De igual forma, en el transcurso de la investigación se identificó como una de las principales debilidades la aglomeración geográfica de los actores en Bucaramanga y su área metropolitana, lo que sugiere que tanto la generación de conocimiento como las diversas actividades productivas, de articulación y financiación se desarrollan específicamente en un área de la región, limitando así el proceso de consolidación del SRI santandereano.

Adicionalmente, en relación a la generación y difusión del conocimiento, la debilidad del SRI se evidencia en el reducido número de investigadores y grupos de investigación presentes en el departamento, así como en la poca indexación de revistas. Lo anterior demuestra la amplia tarea por realizar en Santander a fin de dinamizar el interés, el flujo y la creación del conocimiento en la región.

Conviene tener en cuenta que si bien Santander cuenta con la participación de importantes actores que contribuyen al desarrollo de la región como lo son: el Parque Tecnológico de Guatiguará, el Centro de Desarrollo Tecnológico de Gas, la Corporación para la Investigación de la Corrosión y la Corporación para la Investigación y Desarrollo en Asfaltos en el Sector Transporte e Industrial, el análisis permitió identificar que en Antioquia existe mayor presencia tanto de Parques Tecnológicos, como de Centros de Desarrollo Tecnológico e Incubadoras, lo cual genera mayor dinamismo en el SRI de Antioquia. 
Por último, se pudo observar que en Santander existe una menor participación de los diferentes actores que conforman el subsistema financiero, situación que constituye una importante debilidad, dado que este subsistema aporta recursos para el desarrollo de los procesos cognitivos, experimentales y productivos que tienen lugar en un Sistema Regional de Innovación.

\section{Conclusiones}

Es pertinente resaltar que si bien el departamento de Santander presenta ciertas fortalezas frente a Antioquia, existen numerosas debilidades, y con ellas un rezago importante en materia de Ciencia, Tecnología e Innovación, CTI, razón por la cual el departamento presenta bajos niveles de inversión en Actividades de CTI. Adicionalmente, el comportamiento inversionista de los subsistemas es desigual y altamente fluctuante. De hecho, en Santander las Instituciones de Educación Superior son quienes jalonan las actividades de CTI. Sin embargo, aún no se logra consolidar la capacidad investigativa del departamento, de modo que logre ser una fortaleza significativa. Por otro lado, el sector productivo del departamento presenta baja participación, lo que hace necesario que el tejido empresarial de Santander fortalezca las acciones en materia de CTI.

Así mismo, es posible observar que el gobierno en su interés por dinamizar las capacidades de CTI, presenta mayor participación en Santander que en Antioquia. Esto sucede debido a que en este último departamento existe una cultura innovadora que permite que los procesos de gestión del conocimiento y de la innovación se desenvuelvan mediante la participación conjunta de todos sus actores. En este orden de ideas, conviene anotar que Santander requiere más espacios para el desarrollo de capacidades de emprendimiento y científico-tecnológicas; el bajo número de Incubadoras de Empresas, Centros de Desarrollo Tecnológico y Parques Tecnológicos evidencian esta situación.
En suma, es posible corroborar que la innovación, como proceso de aprendizaje colaborativo, demanda la participación de todos los actores del SRI. Antioquia, al contar con contribuciones relevantes y poco fluctuantes en los comportamientos de todos sus subsistemas, evidencia la superioridad en materia de CTI frente al departamento de Santander. Cabe destacar que el SRI de Antioquia es modelo a nivel mundial, evidencia de ello es su participación en el Foro Mundial de Ecosistemas de Innovación realizado en Sillicon Valley, donde mostró la apuesta que hacen por la innovación ante empresarios, científicos, académicos y emprendedores del mundo (Álvarez, 2015).

Finalmente, conviene resaltar que es trascendental que los actores del SRI de Santander dirijan la mirada al sinnúmero de experiencias positivas alrededor del mundo, de regiones que han obtenido un crecimiento económico sostenido gracias al fortalecimiento en las capacidades de CTI. De igual manera, es esencial que las diversas instituciones que integran el SRI de Santander fortalezcan la interacción entre ellas, consoliden una efectiva cultura innovadora y aumenten la contribución en las inversiones en ACTI; de modo que la actividad innovadora del departamento sea llevada a cabo por todos los subsistemas de forma articulada, puesto que a medida que se consoliden mayores dinámicas en CTI los incrementos en la productividad y competitividad departamental serán más significativos.

\section{Agradecimientos}

Los autores agradecen a la Vicerrectoría de Investigación y Extensión de la Universidad Industrial de Santander, por la financiación del proyecto de investigación: Impacto del Sistema Regional de Innovación de Santander en la dinámica productiva de los Sectores Apuesta del departamento, con código 1765. Igualmente agradecen al grupo de investigación Estudios en Microeconomía Aplicada y Regulación, EMAR, adscrito a la Escuela de Economía y Administración de la UIS. 


\section{Referencias}

Álvarez, V. (18 de febrero de 2015). Modelo de innovación de Medellín será expuesto en Silicon Valley: La transformación de la ciudad sigue generando admiración. El Tiempo. Recuperado de http://www.eltiempo.com/colombia/medellin/ modelo-de-innovacion-de-medellin/15263660

Arenas-Díaz, P., et al. (2013). Plan Estratégico Departamental de Ciencia, Tecnología e Innovación - PEDCTI Santander 2020. Recuperado de: http://www. colciencias.gov.co/sites/default/files/upload/paginas/pedcti-santander.pdf

Arias-Manjarrez, C.C., Arenas-Díaz, P., Carrillo-Zambrano, E., \& Flórez-Gómez, L.Y. (2013). Aproximación al Sistema Regional de Ciencia, Tecnología e Innovación del departamento de Santander. Gerencia, Tecnología e información, 12 (34), 45-58. Recuperado de http://revistas.uis.edu.co/index.php/ revistagti/article/view/3845

Baena, E., Sánchez, J.J., \& Montoya, O. (2006). Importancia de los Centros de Desarrollo Productivo en las regiones: el caso del Centro de Desarrollo Productivo (CDP) de las confecciones de Risaralda (Colombia). Scientia Et Technica, 12 (31), 171-176. Recuperado de http://revistas.utp.edu.co/index. php/revistaciencia/article/view/6421

Cámara de Comercio de Bucaramanga. (2014). Factor Innovación y Desarrollo: marcas concedidas en Colombia 2013. Informes de competitividad, (19), 1. Recuperado de: http://www.compite360.com/ getattachment/142f8ef6-ef14-4b12-b5f9-f560a$34238 \mathrm{ba} / F a c t o r-I n n o v a c i o n-y-D e s a r r o l l o-M a r-$ cas-en-Colombia;;.aspx

Cámara de Comercio de Bucaramanga. (2015). Constitución de empresas Santander 2014. Actualidad económica, (116), 1. Recuperado de: http:// www.camaradirecta.com/temas/documentos $\% 20$ pdf/informes\%20de\%20actualidad/2015/Constituidas\%202014.pdf
Claudia Patricia Cote-Peña Claudia Patricia Meneses-Amaya Cristian Johan Arenas-Morantes

Danitza Ivonne Caballero-Pérez

Camargo, M. (01 de marzo de 2013). Medellín, la ciudad más innovadora del mundo. Revista Semana. Recuperado de http://www.semana.com/ nacion/articulo/medellin-ciudad-mas-innovadora-del-mundo/334982-3

Canever, M.D., \& Carraro, A. (2012). Creación de empresas y recuperación económica: El caso de Rio Grande do Sul. Revista CEPAL, (108), 133-146. Recuperado de: http://repositorio.cepal.org/bitstream/handle/11362/11563/108133146_es.pdf

Doing Businnes Subnational. (2013). Doing Business en Colombia 2013. Regulaciones inteligentes para las pequeñas y medianas empresas. Washington, Estados Unidos: Banco Mundial.

Fernández-de Lucio, I., \& Castro-Martínez, E. (1995). La nueva política de articulación del sistema de innovación de España. VI Seminario Latinoamericano de Gestión Tecnológica. Seminario llevado a cabo en Concepción, Chile.

Llisterri, J.J, \& Pietrobelli, C. (Ed). (2011). Los sistemas regionales de innovación en América Latina. Washington, Estados Unidos: Banco Interamericano de Desarrollo.

Navarro-Arancegui, M., Franco-Rodríguez, S., Murciego-Alonso, A., \& Gibaja-Martíns, J.J. (2012). Metodología de benchmarking territorial: la necesidad de identificación de las regiones de referencia. ICE Innovación y Competitividad, (869), 115132. Recuperado de: http://www.revistasice.com/ CachePDF/ICE_869_115-132_E080E6A6CC5D2C375FB065E281689CB5.pdf

Observatorio Colombiano de Ciencia y Tecnología [OCyT]. (2014a). Inversión en ACTI e I+D. Noticias OCyT. Recuperado de http://ocyt.org.co/es-es/noticias/ArtMID/478/ArticleID/216/Inversi243n-enACTI-e-ID

Observatorio Colombiano de Ciencia y Tecnología [OCyT]. (2014b). Informe Anual de Indicadores de 
Ciencia y Tecnología. Bogotá, Colombia: Observatorio Colombiano de Ciencia y Tecnología.

Oquendo-Gómez, A. F., \& Acevedo-Álvarez, C. A. (2012). El Sistema de Innovación Colombiano: fundamentos, dinámicas y avatares. Revista Trilogía, (6), 105-120. Recuperado de: http://itmojs.itm. edu.co/index.php/trilogia/article/view/254

Organización para la Cooperación y Desarrollo Económicos [OCDE]. (2003). Manual de Frascati: propuesta de norma práctica para encuestas de investigación y desarrollo experimental. París, Francia: Fundación Española para la Ciencia y la Tecnología.

Palacios-Preciado, M., \& Duque-Oliva, E.J. (2011). Modelos de negocio: propuesta de un marco conceptual para centros de productividad. Adminis- tración \& Desarrollo, 39 (53), 23-34. Recuperado de: https://dialnet.unirioja.es/servlet/articulo?codigo $=3776508$

Pinilla, M., Camacho, J., Guerrero, A., Pradilla, H., \& Cote, C. (2001). Desarrollo regional: propuestas y desafíos. Bucaramanga, Colombia: Centro de Estudios Regionales CER- UIS, SIC.

Ramírez, J., \& De Aguas, J. (2015). Escalafón de la competitividad de los departamentos de Colombia 2015. Versión en edición. Colombia: CEPAL.

Zuluaga, D. (2006). Benchmarking como herramienta de evaluación y diagnóstico de sistemas de innovación. I Congreso Iberoamericano de Ciencia, Tecnología, Sociedad e Innovación CTS+l. Congreso llevado a cabo en México D.F., México. 